\title{
Harm perceptions of waterpipe tobacco smoking among university students in five Eastern Mediterranean Region countries: A cross-sectional study
}

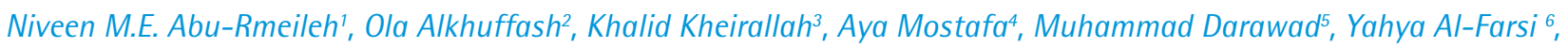 \\ Afzalhussein Yusufali ${ }^{7}$, Justin Thomas ${ }^{8}$, Mohamed Salama $^{9}$, Randah R. Hamadeh ${ }^{10}$, Rima Nakkash ${ }^{11}$, Ramzi G. Salloum ${ }^{12}$
}

\section{ABSTRACT}

INTRODUCTION Waterpipe tobacco smoking (WTS) continues to be very common in the Eastern Mediterranean Region (EMR), partially because of cultural acceptance but also because of misconceptions of its harm. This paper aimed to describe the beliefs towards waterpipe harm of university students who smoked waterpipe in five EMR countries.

METHODS This study was conducted in 2016 across five EMR countries: Egypt, Jordan, Occupied Palestinian Territories, Oman and United Arab Emirates (UAE). Participants were recruited from among university students in each country. Students' characteristics, smoking behavior, flavor preference and knowledge of WTS harm were collected using an internet-based survey. Participants were included if they were ever waterpipe tobacco smokers and between 18 and 29 years of age. Bivariate analyses assessed variations in student-perceived WTS harm across the countries. Linear regression analysis was used to assess WTS perceived harm differences between students in the different countries. RESULTS A total of 2544 university students participated from the five countries. Among ever smoking students, $66 \%$ reported WTS in the past 30 days, with the highest proportions (40\%) from Occupied Palestinian Territories (OPT) and (41\%) Jordan. Dual smoking of waterpipe and cigarettes was highest among students from Egypt. Most participants from the five countries had high level of perceived harm related to WTS during pregnancy. Less than $50 \%$ of the students believed that WTS could lead to the death of the smoker, can be harmful for non-smokers and have an addictive effect. Female students, those older than 22 years, and those who didn't smoke waterpipe in the last 30 days significantly had a higher level of WTS perceived harm. Participating students believed that cigarettes are more addictive and contain more nicotine compared to waterpipe.

CONCLUSIONS Misperceptions of waterpipe harm are common among university students in the five EMR countries. Immediate public health action is needed, including enforcement of waterpipe tobacco control regulations along with awareness campaigns.

\author{
AFFILIATION \\ 1 Institute of Community and Public Health, \\ Birzeit University, Occupied Palestinian \\ Territories \\ 2 Statistic Department, Hacettepe University, \\ Ankara, Turkey \\ 3 Department of Public Health, Medical \\ School, Jordan University of Science and \\ Technology, Irbid, Jordan \\ 4 Department of Community Environmental \\ and Occupational Medicine, Faculty of \\ Medicine, Ain Shams University, Cairo, Egypt \\ 5 The University of Jordan, Amman, Jordan \\ 6 Sultan Qaboos University, Muscat, Oman \\ 7 Dubai Medical College, Dubai, United Arab \\ Emirates \\ 8 Zayed University, Abu Dhabi, United Arab \\ Emirates \\ 9 Mansoura University, Mansoura, Egypt \\ 10 College of Medicine and Medical Sciences, \\ Arabian Gulf University, Manama, Bahrain \\ 11 Faculty of Health Sciences, American \\ University of Beirut, Beirut, Lebanon \\ 12 Department of Health Outcomes and \\ Policy, College of Medicine, University of \\ Florida, Gainesville, Florida, United States \\ CORRESPONDENCE TO \\ Niveen M.E. Abu-Rmeileh. Institute of \\ Community and Public Health, Birzeit \\ University, Occupied Palestinian Territories. \\ E-mail:nrmeileh@birzeit.edu
}

\section{KEYWORDS}

waterpipe, Eastern Mediterranean, harm perception, university students

Received: 13 March 2018

Revised: 10 April 2018

Accepted: 16 April 2018
INTRODUCTION

The Eastern Mediterranean Region (EMR) has some of the highest prevalence rates of waterpipe tobacco smoking (WTS) and these rates have been increasing 
over the past two decades ${ }^{1-8}$. In this region, it is culturally more acceptable for young people to smoke waterpipe than cigarettes. In fact, sharing waterpipe with family members is often how WTS is initiated among the young ${ }^{9-11}$. In most EMR countries, prevalence rates of WTS are slightly higher in boys. The gender gap in prevalence rates is much larger, however, for cigarette smoking ${ }^{12,13}$. Such gender differences were attributed to the social acceptability of WTS, especially among girls, compared to cigarette smoking ${ }^{14}$. Some studies have shown that, among youths in most of the EMR, waterpipe has replaced cigarettes as the most common method of tobacco use ${ }^{15}$.

A review of attitude, beliefs and perceived WTS harm has indicated that waterpipe smokers perceive it to be less harmful than cigarettes and so more likely to continue to smoke waterpipe ${ }^{16}$. These studies were limited to few constructs and they have not been compared across EMR countries that varied in WTS prevalence. Given the continuous increase in WTS, especially among young people in the Eastern Mediterranean countries and the limited studies, we aimed to assess the level of knowledge of WTS harm among university students in five EMR countries.

\section{METHODS}

A cross-sectional study was conducted in 2016 across five EMR countries: Egypt, Jordan, Occupied Palestinian Territories, the United Arab Emirates (UAE) and Oman. The target population for the study was ever waterpipe smokers among young adults (18-29 years old) enrolled in universities in the selected countries. The participating universities were: Birzeit University (13 963), Jordan University of Science and Technology (20 000), University of Jordan (37 692), Sultan Qaboos University (15 357), Dubai Medical College (500), Zayed University (9 217), Mansoura University (91 041) and Ain Shams University (168 970).

Subjects were selected using convenience samples, with the purpose of recruiting relatively heterogeneous samples involving participants from key population groups (i.e. undergraduate vs graduate, and male vs female). Initial invitations were sent to all students through e-mail, Facebook, and students' university portals inviting them to participate in the WTS survey with the aim of reaching a minimum of 400 students from each country. The estimated sample size for a proportion sample of a finite population with 95\% Confidence Interval (CI) and assumption of 50\% prevalence was 384 students. If the target size of 400 respondents was reached at a site before the end of the project, data collection continued to increase the sample size and hence power.

The email messages provided links to the internetbased survey. Participants were assured of the confidentiality of the survey, that their responses would remain anonymous and that their smoking status would not be made public, and finally were informed that they could leave the survey at any time.

The questionnaire used was adopted from a standard survey of university-based WTS users and measured basic demographic characteristics, WTS history, current use, attitudes and perceptions regarding WTS, and the concurrent use of other tobacco products. The health warning labels were pretested in qualitative interviews and the questionnaires were pre-piloted in each country. The questionnaire was translated into Arabic by two translators, and then reviewed and revised for nuances in dialect by the reseach team in each country.

Current waterpipe smokers and current cigarette smokers were defined as those who smoked in the last 30 days. Age, gender, WTS initiation location and perceived harm knowledge variables were measured for each country. Perceptions of harm were measured using 9 questions based on the following health warnings: WTS is addictive; waterpipe smoke can harm children; WTS causes fatal lung disease; WTS causes cancer; WTS causes strokes and heart disease; WTS during pregnancy can harm the baby; WTS can kill you; waterpipe smoke causes fatal lung disease in non-smokers; and quitting WTS now greatly reduces serious risks to your health. Each question had answers that were grouped into four codes: 'Not at all/Little' (1), 'Somewhat' (2), 'A lot/ Completely' (3), and 'Don't know' (4). For each participant, mean perceived harm was calculated using the nine above questions. The distribution of the WTS harm was tested for normality. Data were presented using numbers/percentages and means/ SD as a appropriate.

Percentages and 95\% CI were calculated for each variable mentioned above. Linear regression was used to assess variations in WTS level of harm beliefs 
between countries after adjusting for age and gender. For all analyses, we defined the level of significance at 0.05 . The statistical analysis was conducted using SPSS version 22. Institutional Review Board approval was obtained from all participating institutions.

\section{RESULTS}

A total of 2544 university students participated: Jordan (745, 29.3\%), OPT (772, 30.3\%), Egypt (728, $28.6 \%)$, UAE $(180,7.1 \%)$ and Oman $(119,4.7 \%)$. Thirty-four per cent of all participants were females. Participants' age ranged between 18 and 29 years, with a mean (SD) of 21.7 (2.8) years. Significant differences in the age and gender by country were detected (for both comparisons $\mathrm{p}<0.001$ ) (Table 1).

The majority of the students (74\%) initiated WTS with friend(s), while $16.5 \%$ initiated it with a family member (Table 1). More than $70 \%$ of the students from Oman, UAE and Egypt started smoking in a café or restaurant, while around $50 \%$ of the students from Jordan and OPT started smoking at home or someone else's home. Very few started WTS at university accommodations (Table 1).

Among waterpipe ever smokers, about two-thirds

Table 1. Study sample characteristics, in percentages (\%)

\begin{tabular}{|c|c|c|c|c|c|c|}
\hline & $\begin{array}{l}\text { Egypt } \\
(\mathrm{N}-728)\end{array}$ & $\begin{array}{l}\text { Jordan } \\
(\mathbf{N}-715)\end{array}$ & $\begin{array}{c}\text { OPT } \\
(\mathbf{N}=\mathbf{7 7 2})\end{array}$ & $\begin{array}{l}\text { Oman } \\
(\mathrm{N}=119)\end{array}$ & $\begin{array}{c}\text { UAE } \\
(\mathrm{N}-180)\end{array}$ & $\begin{array}{c}\text { Total } \\
(\mathrm{N}=2511)\end{array}$ \\
\hline \multicolumn{7}{|l|}{ Gender } \\
\hline \multirow[t]{2}{*}{ Male } & 88.6 & 63.3 & 48.6 & 91.0 & 50.8 & 65.6 \\
\hline & $(86.0-91.1)$ & $(59.8-66.7)$ & $(45.1-52.2)$ & $(85.7-96.3)$ & $(42.2-59.3)$ & $(63.6-67.5)$ \\
\hline \multirow[t]{2}{*}{ Female } & 11.4 & 36.7 & 51.4 & 9.0 & 49.2 & 34.4 \\
\hline & $(8.9-14.0)$ & $(33.3-40.2)$ & $(47.8-54.9)$ & $(3.7-14.3)$ & $(40.7-57.8)$ & $(32.5-36.4)$ \\
\hline \multicolumn{7}{|l|}{ Age } \\
\hline \multirow[t]{2}{*}{ Young (18-22 years) } & 30.8 & 76.9 & 84.1 & 34.5 & 59.4 & 62.7 \\
\hline & $(27.4-34.1)$ & $(73.9-79.9)$ & $(81.5-86.6)$ & $(25.9-43.0)$ & $(52.3-66.6)$ & $(60.8-64.5)$ \\
\hline \multirow[t]{2}{*}{ Old ( 23 years or more) } & 69.2 & 23.1 & 15.9 & 65.5 & 40.6 & 37.3 \\
\hline & $(65.9-72.6)$ & $(20.1-26.1)$ & $(13.4-18.5)$ & $(57.0-74.1)$ & $(33.4-47.7)$ & $(35.5-39.2)$ \\
\hline Mean & $23.8(2.86)$ & $20.9(2.20)$ & $20.6(2.19)$ & $23.6(2.93)$ & $20.8(2.45)$ & $21.7(2.80)$ \\
\hline \multicolumn{7}{|l|}{ Marital status } \\
\hline \multirow[t]{2}{*}{ Single } & 77.9 & 96.9 & 94.9 & 75.0 & 94.4 & 90.2 \\
\hline & $(74.6-81.2)$ & $(95.6-98.1)$ & $(93.3-96.4)$ & $(67.1-82.9)$ & (91.0-97.8) & (89.0-91.4) \\
\hline \multirow[t]{2}{*}{ Married } & 22.1 & 3.1 & 5.1 & 25.0 & 5.6 & 9.8 \\
\hline & $(18.8-25.4)$ & $(1.9-4.4)$ & $(3.6-6.7)$ & $(17.1-32.9)$ & $(2.2-9.0)$ & $(8.6-11.0)$ \\
\hline \multicolumn{7}{|l|}{ Employment status } \\
\hline \multirow[t]{2}{*}{ Unemployed } & 42.1 & 73.6 & 69.1 & 54.5 & 73.5 & 63.3 \\
\hline & $(37.9-46.3)$ & $(70.3-76.9)$ & $(65.6-72.6)$ & $(45.2-63.9)$ & $(66.0-81.0)$ & $(61.2-65.3)$ \\
\hline \multirow[t]{2}{*}{ Employed } & 57.9 & 26.4 & 30.9 & 45.5 & 26.5 & 36.7 \\
\hline & $(53.7-62.1)$ & $(23.1-29.7)$ & $(27.4-34.4)$ & $(36.1-54.8)$ & $(19.0-34.0)$ & $(34.7-38.8)$ \\
\hline \multicolumn{7}{|c|}{$\begin{array}{l}\text { Current waterpipe smoker (smoke in } \\
\text { the past } 30 \text { days) }\end{array}$} \\
\hline \multirow[t]{2}{*}{ No } & 26.2 & 31.5 & 36.8 & 46.1 & 47.8 & 33.8 \\
\hline & $(22.7-29.7)$ & $(28.1-34.8)$ & $(33.3-40.2)$ & $(37.0-55.2)$ & $(40.4-55.1)$ & $(31.9-35.7)$ \\
\hline \multirow[t]{2}{*}{ Yes } & 73.8 & 68.5 & 63.2 & 53.9 & 52.2 & 66.2 \\
\hline & $(70.3-77.3)$ & $(65.2-71.9)$ & $(59.8-66.7)$ & $(44.8-63.0)$ & $(44.9-59.6)$ & $(64.3-68.1)$ \\
\hline \multicolumn{7}{|c|}{$\begin{array}{l}\text { With whom did you first smoke } \\
\text { waterpipe? }\end{array}$} \\
\hline \multirow[t]{2}{*}{ Alone/no one } & 21.6 & 6.9 & 5.0 & 7.1 & 3.5 & 10.1 \\
\hline & $(18.4-24.8)$ & $(5.0-8.8)$ & $(3.4-6.6)$ & $(2.4-11.8)$ & $(0.8-6.3)$ & $(8.9-11.3)$ \\
\hline \multirow[t]{2}{*}{ With a friend } & 42.7 & 34.3 & 30.3 & 54.0 & 32.2 & 36.2 \\
\hline & $(38.9-46.6)$ & $(30.8-37.9)$ & $(26.9-33.6)$ & $(44.8-63.2)$ & $(25.2-39.2)$ & $(34.2-38.1)$ \\
\hline With several friends & 34.9 & 40.5 & 33.8 & 34.5 & 49.7 & 37.3 \\
\hline
\end{tabular}


Table 1. Continued

\begin{tabular}{|c|c|c|c|c|c|c|}
\hline & $\begin{array}{l}\text { Egypt } \\
\text { (N 728) }\end{array}$ & $\begin{array}{l}\text { Jordan } \\
(\mathrm{N} \text { 715) }\end{array}$ & $\begin{array}{c}\text { OPT } \\
(\mathrm{N} \text { 772) }\end{array}$ & $\begin{array}{c}\text { Oman } \\
(\mathrm{N}-119)\end{array}$ & $\begin{array}{c}\text { UAE } \\
(\mathrm{N}=180)\end{array}$ & $\begin{array}{l}\text { Total } \\
(\mathrm{N}=25 \text { (1) }\end{array}$ \\
\hline & $(31.2-38.6)$ & $(36.9-44.2)$ & $(30.3-37.2)$ & $(25.7-43.3)$ & $(42.2-57.2)$ & $(35.3-39.2)$ \\
\hline \multirow[t]{2}{*}{ With family members } & 0.8 & 18.2 & 31.0 & 4.4 & 14.6 & 16.5 \\
\hline & $(0.1-1.5)$ & $(15.4-21.1)$ & $(27.6-34.3)$ & $(0.6-8.2)$ & $(9.3-19.9)$ & $(15.0-18.0)$ \\
\hline \multicolumn{7}{|l|}{ Where did you first smoke waterpipe? } \\
\hline \multirow[t]{2}{*}{ Cafe/restaurant } & 81.4 & 51.8 & 39.9 & 74.3 & 72.3 & 58.9 \\
\hline & $(78.4-84.4)$ & $(48.1-55.6)$ & $(36.3-43.5)$ & $(65.7-82.8)$ & $(65.5-79.1)$ & $(56.9-60.9)$ \\
\hline \multirow{2}{*}{$\begin{array}{l}\text { Smoke shop e.g. commercial waterpipe } \\
\text { establishment }\end{array}$} & 3.0 & 1.5 & 0.4 & 11.9 & 7.8 & 2.5 \\
\hline & $(1.7-4.3)$ & $(0.6-2.4)$ & & $(5.6-18.2)$ & $(3.7-11.9)$ & $(1.9-3.1)$ \\
\hline \multirow[t]{2}{*}{ At home } & 3.3 & 20.3 & 34.3 & 3.0 & 10.2 & 18.4 \\
\hline & $(1.9-4.7)$ & $(17.3-23.4)$ & $(30.8-37.9)$ & & $(5.6-14.9)$ & $(16.8-20.0)$ \\
\hline \multirow[t]{2}{*}{ Someone else's home } & 8.3 & 25.5 & 22.7 & 7.9 & 9.0 & 17.9 \\
\hline & $(6.2-10.5)$ & (22.2-28.8) & (19.6-25.9) & $(2.7-13.2)$ & $(4.7-13.4)$ & $(16.3-19.5)$ \\
\hline \multirow[t]{2}{*}{ University accommodations } & 3.9 & 0.9 & 2.6 & 3.0 & 0.6 & 2.3 \\
\hline & $(2.4-5.4)$ & $(0.2-1.6)$ & $(1.4-3.7)$ & & & $(1.7-2.9)$ \\
\hline
\end{tabular}

of participants reported WTS within the last 30-days (66.2\%, 95\% CI: 64.3-68.1\%) (Figure 1). The overall percentage of current cigarette-only smokers was $12 \%$, while the overall dual (waterpipe and cigarette) smoking percentage was $30 \%$.

Table 2 presents university students' perceived WTS harm among ever waterpipe smokers. Overall, $72 \%$ of all students believed that waterpipe smoking during pregnancy can harm the baby, $73 \%$ believed that WTS can be harmful to children, $62 \%$ believed that WTS can cause fatal lung disease and $60 \%$ believed that quitting WTS can reduce serious risk to health. Around $50 \%$ of all students believed that
Figure 1. The percentages of current waterpipe tobacco smokers, current cigarette smokers and dual smokers, among university students in the EMR

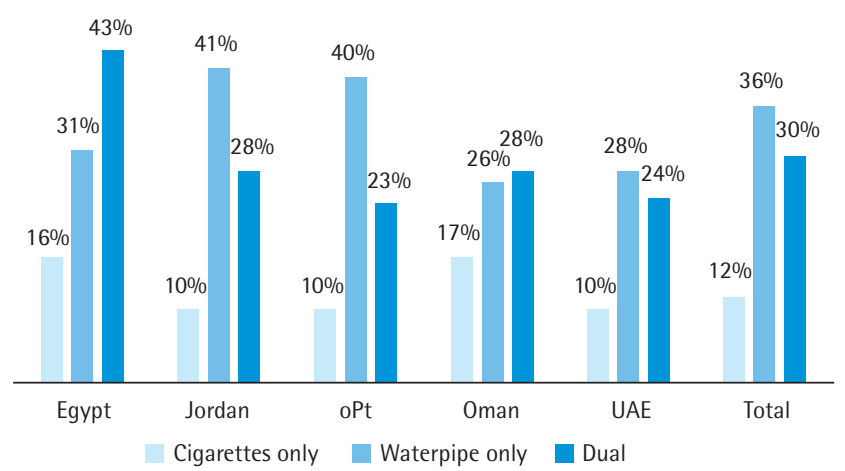

Table 2. University students' perceived WTS harm among ever waterpipe smokers in five EMR countries, in percentages $(\%)$

\begin{tabular}{|c|c|c|c|c|c|c|}
\hline & Egypt & Jordan & OPT & Oman & UAE & Total \\
\hline \multicolumn{7}{|l|}{$\begin{array}{l}\text { Do you believe the following } \\
\text { statements? }\end{array}$} \\
\hline Waterpipe smoking is addictive & $(\mathrm{N}=620)$ & $(\mathrm{N}=739)$ & $(\mathrm{N}=758)$ & $(\mathrm{N}=116)$ & $(\mathrm{N}=178)$ & $(\mathrm{N}=2401)$ \\
\hline \multirow[t]{2}{*}{ Not at all/Little } & 53.7 & 44.7 & 39.0 & 34.0 & 39.9 & 44.4 \\
\hline & $(49.8-57.6)$ & $(41.1-48.3)$ & $(35.4-42.4)$ & $(25.8-43.1)$ & $(32.7-47.1)$ & $(42.4-46.3)$ \\
\hline \multirow[t]{2}{*}{ Somewhat } & 21.8 & 25.4 & 23.4 & 24.1 & 23.6 & 23.6 \\
\hline & $(18.5-25.0)$ & $(22.2-28.5)$ & $(20.3-26.4)$ & $(16.4-31.9)$ & $(17.4-29.8)$ & $(21.9-25.3)$ \\
\hline \multirow[t]{2}{*}{ A Lot/Completely } & 23.2 & 27.4 & 36.4 & 41.4 & 35.4 & 30.4 \\
\hline & $(19.9-26.5)$ & $(24.2-30.7)$ & $(33.0-39.8)$ & $(32.4-50.3)$ & $(28.4-42.4)$ & $(28.6-32.3)$ \\
\hline \multirow[t]{2}{*}{ Don't know } & 1.3 & 2.5 & 1.3 & 0 & 1.1 & 1.6 \\
\hline & $(0.4-2.2)$ & $(1.3-3.6)$ & $(0.5-2.1)$ & & $(-0.4-2.7)$ & $(1.1-2.1)$ \\
\hline
\end{tabular}


Table 2. continued

\begin{tabular}{|c|c|c|c|c|c|c|}
\hline & Egypt* & Jordan & OPT & Oman & UAE & Total \\
\hline Waterpipe smoke can harm children & $(\mathrm{N}=646)$ & $(\mathrm{N}=732)$ & $(\mathrm{N}=755)$ & $(N=114)$ & $(\mathrm{N}=177)$ & $(\mathrm{N}=2424)$ \\
\hline \multirow[t]{2}{*}{ Not at all/Little } & 24.5 & 10.7 & 6.2 & 15.8 & 10.7 & 13.2 \\
\hline & $(21.1-27.8)$ & $(8.4-12.9)$ & $(4.5-7.9)$ & $(9.1-22.5)$ & $(6.2-15.3)$ & $(11.9-14.5)$ \\
\hline \multirow[t]{2}{*}{ Somewhat } & 15.9 & 8.6 & 11.9 & 16.7 & 8.5 & 12.0 \\
\hline & $(13.1-18.8)$ & $(6.6-10.6)$ & $(9.6-14.2)$ & $(9.8-23.5)$ & $(4.4-12.6)$ & $(10.7-13.3)$ \\
\hline \multirow[t]{2}{*}{ A Lot/Completely } & 58.2 & 78.4 & 81.2 & 67.5 & 78.5 & 73.4 \\
\hline & $(54.4-62.0)$ & $(75.6-81.5)$ & $(78.4-84.0)$ & $(58.9-76.1)$ & $(72.5-84.6)$ & $(71.7-75.2)$ \\
\hline \multirow[t]{2}{*}{ Don't know } & 1.4 & 2.2 & 0.73 & 0 & 2.3 & 1.4 \\
\hline & $(0.5-2.3)$ & $(1.1-3.2)$ & $(0.1-1.2)$ & & $(014-4.4)$ & $(0.9-1.9)$ \\
\hline $\begin{array}{l}\text { Waterpipe smoking causes fatal lung } \\
\text { disease }\end{array}$ & $(\mathrm{N}=633)$ & $(\mathrm{N}=735)$ & $(\mathrm{N}=756)$ & $(\mathrm{N}=114)$ & $(\mathrm{N}=178)$ & $(\mathrm{N}=2416)$ \\
\hline \multirow[t]{2}{*}{ Not at all/Little } & 27.3 & 13.7 & 8.9 & 13.2 & 10.1 & 15.5 \\
\hline & $(23.9-30.8)$ & $(11.3-16.2)$ & $(6.8-10.9)$ & $(7.0-19.4)$ & $(5.7-14.5)$ & $(14.0-16.9)$ \\
\hline \multirow[t]{2}{*}{ Somewhat } & 20.4 & 16.5 & 19.7 & 25.4 & 15.2 & 18.8 \\
\hline & $(17.2-23.5)$ & $(13.8-19.1)$ & $(16.9-22.5)$ & $(17.4-33.4)$ & $(9.9-20.4)$ & $(17.3-20.4)$ \\
\hline \multirow[t]{2}{*}{ A Lot/Completely } & 49.0 & 66.4 & 68.0 & 61.4 & 68.0 & 62.3 \\
\hline & $(45.1-52.9)$ & $(63.0-69.8)$ & $(65.1-71.7)$ & $(52.5-70.3)$ & $(61.1-74.8)$ & $(60.4-64.3)$ \\
\hline \multirow[t]{2}{*}{ Don't know } & 3.3 & 3.4 & 3.0 & 0 & 6.7 & 3.4 \\
\hline & $(1.9-4.7)$ & $(2.1-4.7)$ & $(1.8-4.3)$ & & $(3.1-10.4)$ & $(2.6-4.1)$ \\
\hline Waterpipe smoking causes cancer & $(\mathrm{N}=602)$ & $(\mathrm{N}=729)$ & $(\mathrm{N}=756)$ & $(\mathrm{N}=115)$ & $(\mathrm{N}=178)$ & $(\mathrm{N}=2380)$ \\
\hline \multirow[t]{2}{*}{ Not at all/Little } & 25.1 & 16.2 & 16.0 & 25.2 & 11.2 & 18.4 \\
\hline & $(21.6-28.5)$ & $(13.5-18.9)$ & $(13.4-18.6)$ & $(17.3-33.2)$ & $(6.6-15.9)$ & $(16.9-20.0)$ \\
\hline \multirow[t]{2}{*}{ Somewhat } & 22.3 & 18.9 & 21.0 & 20.2 & 12.9 & 20.0 \\
\hline & $(19.0-25.5)$ & $(16.1-21.8)$ & $(18.1-23.9)$ & $(12.8-27.5)$ & $(8.0-17.8)$ & $(18.4-21.6)$ \\
\hline \multirow[t]{2}{*}{ A Lot/Completely } & 44.5 & 56.0 & 56.6 & 55.3 & 66.9 & 54.0 \\
\hline & $(40.6-48.4)$ & $(52.4-59.6)$ & $(53.1-60.1)$ & $(46.1-64.4)$ & $(59.9-73.8)$ & $(52.0-56.0)$ \\
\hline \multirow[t]{2}{*}{ Don't know } & 8.1 & 8.9 & 6.3 & 0 & 9.0 & 7.5 \\
\hline & $(6.0-10.3)$ & $(6.8-11.0)$ & $(4.6-8.1)$ & & $(4.8-13.2)$ & $(6.4-8.5)$ \\
\hline $\begin{array}{l}\text { Waterpipe smoking causes strokes and } \\
\text { heart disease }\end{array}$ & $(\mathrm{N}=630)$ & $(\mathrm{N}=729)$ & $(\mathrm{N}=752)$ & $(\mathrm{N}=114)$ & $(\mathrm{N}=178)$ & $(\mathrm{N}=2403)$ \\
\hline \multirow[t]{2}{*}{ Not at all/Little } & 33.2 & 15.0 & 16.0 & 24.6 & 15.2 & 20.5 \\
\hline & $(29.5-36.9)$ & $(12.4-17.5)$ & $(13.3-18.6)$ & $(16.7-32.5)$ & $(1.6-28.7)$ & $(18.9-22.1)$ \\
\hline \multirow[t]{2}{*}{ Somewhat } & 18.7 & 19.1 & 19.8 & 21.1 & 17.4 & 19.2 \\
\hline & $(15.7-21.8)$ & $(16.2-21.9)$ & $(17.0-22.7)$ & $(13.6-28.5)$ & $(3.1-31.7)$ & $(17.6-20.8)$ \\
\hline \multirow[t]{2}{*}{ A Lot/Completely } & 41.4 & 56.4 & 58.4 & 54.4 & 59.0 & 53. \\
\hline & $(37.6-45.3)$ & $(52.8-60.0)$ & $(54.9-61.9)$ & $(45.2-63.5)$ & $(40.4-77.5)$ & $(51.2-55.2)$ \\
\hline \multirow[t]{2}{*}{ Don't know } & 6.7 & 9.6 & 5.9 & 0 & 8.4 & 7.1 \\
\hline & $(4.7-8.6)$ & $(7.5-11.7)$ & $(4.2-7.5)$ & & $(-2.1-18.9)$ & $(6.1-8.1)$ \\
\hline $\begin{array}{l}\text { Waterpipe smoking during pregnancy } \\
\text { can harm the baby }\end{array}$ & $(\mathrm{N}=630)$ & $(\mathrm{N}=730)$ & $(\mathrm{N}=756)$ & $(\mathrm{N}=108)$ & $(\mathrm{N}=177)$ & $(\mathrm{N}=2401)$ \\
\hline \multirow[t]{2}{*}{ Not at all/Little } & 22.9 & 10.0 & 5.8 & 16.7 & 7.3 & 12.2 \\
\hline & $(19.6-26.1)$ & $(7.8-12.2)$ & $(4.2-7.5)$ & $(9.6-23.7)$ & $(3.5-11.2)$ & $(10.9-13.5)$ \\
\hline \multirow[t]{2}{*}{ Somewhat } & 14.9 & 8.8 & 9.7 & 15.7 & 7.9 & 10.9 \\
\hline & $(12.1-17.7)$ & $(6.7-10.8)$ & $(7.6-11.8)$ & $(8.9-22.6)$ & $(3.9-11.9)$ & $(9.7-12.2)$ \\
\hline \multirow[t]{2}{*}{ A Lot/Completely } & 57.5 & 74.5 & 81.5 & 67.8 & 78.5 & 72.2 \\
\hline & $(53.6-61.3)$ & $(71.4-77.7)$ & (78.7-84.3) & $(58.8-76.4)$ & $(72.5-84.6)$ & $(70.4-74.0)$ \\
\hline \multirow[t]{2}{*}{ Don't know } & 4.8 & 6.7 & 3.0 & 0 & 6.2 & 4.7 \\
\hline & $(3.1-6.4)$ & $(4.9-8.5)$ & $(1.8-4.3)$ & & $(2.7-9.8)$ & $(3.9-5.6)$ \\
\hline
\end{tabular}


Table 2. continued

\begin{tabular}{|c|c|c|c|c|c|c|}
\hline & Egypt* & Jordan & OPT & Oman & UAE & Total \\
\hline Waterpipe smoking can kill you & $(\mathrm{N}=604)$ & $(\mathrm{N}=729)$ & $(\mathrm{N}=753)$ & $(\mathrm{N}=114)$ & $(\mathrm{N}=177)$ & $(\mathrm{N}=2377)$ \\
\hline \multirow[t]{2}{*}{ Not at all/Little } & 37.9 & 22.6 & 24.3 & 21.9 & 21.5 & 26.9 \\
\hline & $(34.0-41.8)$ & $(19.6-25.7)$ & $(21.2-27.4)$ & $(14.3-29.5)$ & $(15.4-27.5)$ & $(25.1-28.7)$ \\
\hline \multirow[t]{2}{*}{ Somewhat } & 17.2 & 19.6 & 20.3 & 25.4 & 18.6 & 19.4 \\
\hline & $(14.2-v 20.2)$ & $(16.7-22.5)$ & $(17.4-23.2)$ & $(17.4-33.4)$ & $(12.9-24.4)$ & $(17.8-21.0)$ \\
\hline \multirow[t]{2}{*}{ A Lot/Completely } & 39.6 & 48.6 & 48.3 & 52.6 & 52.6 & 46.7 \\
\hline & $(35.7-43.5)$ & $(44.9-52.2)$ & $(44.8-51.9))$ & $(43.5-61.8)$ & $(45.2-59.9)$ & $(44.7-48.7)$ \\
\hline \multirow[t]{2}{*}{ Don't know } & 5.3 & 9.2 & 7.0 & 0 & 7.3 & 6.9 \\
\hline & $(3.5-7.1)$ & $(7.1-11.3)$ & $(5.2-8.9)$ & & $(3.5-7.1)$ & $(5.9-8.0)$ \\
\hline $\begin{array}{l}\text { Waterpipe smoke causes fatal lung } \\
\text { disease in nonsmokers }\end{array}$ & $(\mathrm{N}=643)$ & $(\mathrm{N}=731)$ & $(N=752)$ & $(\mathrm{N}=115)$ & $(\mathrm{N}=177)$ & $(\mathrm{N}=2418)$ \\
\hline \multirow[t]{2}{*}{ Not at all/Little } & 35.8 & 21.1 & 24.1 & 20.9 & 22.0 & 26.0 \\
\hline & $(32.1-39.5)$ & (18.1-24.0) & $(21.0-27.1)$ & (13.4-28.3) & $(15.9-28.1)$ & $(24.2-27.7)$ \\
\hline \multirow[t]{2}{*}{ Somewhat } & 15.9 & 21.6 & 22.9 & 39.6 & 17.5 & 20.6 \\
\hline & $(13.0-18.7)$ & (18.6-24.6) & $(19.9-25.9)$ & $(21.2-37.9)$ & $(11.9-23.1)$ & $(18.9-22.2)$ \\
\hline \multirow[t]{2}{*}{ A Lot/Completely } & 43.5 & 46.8 & 45.2 & 49.6 & 49.6 & 45.8 \\
\hline & (39.7-47.4 & (43.2-50.4) & (41.7-48.8) & $(40.4-58.7)$ & $(42.9-57.6)$ & $(43.8-47.8)$ \\
\hline \multirow[t]{2}{*}{ Don't know } & 4.8 & 10.2 & 7.8 & 0 & 10.2 & 7.7 \\
\hline & $(3.2-6.5)$ & $(8.3-12.8)$ & $(5.9-9.8)$ & & $(5.7-14.6)$ & $(6.6-8.7)$ \\
\hline $\begin{array}{l}\text { Quitting Waterpipe smoking now } \\
\text { greatly reduces serious risks to your } \\
\text { health }\end{array}$ & $(\mathrm{N}=643)$ & $(\mathrm{N}=730)$ & $(\mathrm{N}=754)$ & $(\mathrm{N}=113)$ & $(\mathrm{N}=179)$ & $(\mathrm{N}=2419)$ \\
\hline \multirow[t]{2}{*}{ Not at all/Little } & 33.6 & 13.7 & 13.8 & 23.0 & 27.4 & 20.5 \\
\hline & $(29.9-37.2)$ & $(11.2-16.2)$ & $(11.3-16.3)$ & (15.2-30.8) & $(20.8-33.9)$ & $(18.9-22.1)$ \\
\hline \multirow[t]{2}{*}{ Somewhat } & 14.0 & 16.8 & 16.9 & 20.9 & 14.7 & 15.6 \\
\hline & $(11.3-16.7)$ & $(12.3-17.5)$ & $(14.2-19.5)$ & $(13.4-28.4)$ & $(9.5-19.9)$ & $(14.1-17.0)$ \\
\hline \multirow[t]{2}{*}{ A Lot/Completely } & 48.8 & 66.1 & 66.8 & 54.5 & 52.5 & 60.3 \\
\hline & $(45.0-52.7)$ & $(62.6-69.5)$ & $(63.5-70.2)$ & $(45.6-64.0)$ & $(45.2-59.9)$ & $(58.3-62.2)$ \\
\hline \multirow[t]{2}{*}{ Don't know } & 3.6 & 5.2 & 2.5 & 0 & 6.2 & 3.8 \\
\hline & $(2.1-5.0)$ & $(3.6-6.8)$ & $(1.4-3.6)$ & & $(2.7-9.8)$ & $(3.0-4.5)$ \\
\hline \multirow[t]{3}{*}{ Mean perceived harm } & $(\mathrm{N}=512)$ & $(\mathrm{N}=704)$ & $(\mathrm{N}=735)$ & $(\mathrm{N}=105)$ & $(\mathrm{N}=173)$ & $(\mathrm{N}=2229)$ \\
\hline & 3.08 & 4.46 & 4.64 & 4.82 & 4.28 & 4.14 \\
\hline & $(2.86-3.29)$ & $(4.26-4.65)$ & $(4.46-4.65)$ & $(4.43-5.21)$ & (3.74-4.83) & $(4.03-4.25)$ \\
\hline
\end{tabular}

* The total number was reported for each variable, as the number of missing was not consistent.

WTS causes cancer, stroke and heart diseases. Less than $50 \%$ of the students believed WTS causes fatal lung disease for non-smokers (46\%), can kill (47\%) and is addictive (30\%). The answers of students from Egypt indicated the lowest level of perceived WTS harm and their answers were significantly different from those of students from the other four countries (Table 2).

Table 3 presents the WTS harm for the five countries by gender, age and smoking status. Overall, female students indicated higher level of perceived WTS harm compared to male students. Only for the question on the effect of quitting smoking there was no significant gender difference. Further, answers from students older than 22 years indicated higher level of perceived WTS harm for the following questions: WTS can harm children, WTS causes fatal lung disease, WTS during pregnancy can harm the baby, and quitting WTS reduces risk to health. Finally, answers from current WTS users indicated lower level of perceived WTS harm compared to non-current smokers for all questions.

When comparing WTS to cigarette, only $11 \%$ of all the students thought that WTS is more addictive compared to $64 \%$ who thought cigarette was more addictive. Seventy-two percent of the Jordanian 
Table 3. University students' perceived WTS harm among ever waterpipe smokers by selected factors, 2016, in percentages $(\%)$

\begin{tabular}{|c|c|c|c|c|c|c|}
\hline & \multicolumn{2}{|c|}{ Gender } & \multicolumn{2}{|c|}{ Current UTS } & \multicolumn{2}{|c|}{ Age } \\
\hline & Vale & Female & Vo & Yes & $18-22$ & $23+$ \\
\hline WTS is addictive & $N(1486)$ & $N(784)$ & $N(776)$ & $N(1557)$ & $N(839)$ & $N(1562)$ \\
\hline Not at all/Little & 48.9 & 35.2 & 33.9 & 48.7 & 46.1 & 43.4 \\
\hline Somewhat & 22.7 & 26.7 & 20.7 & 25.5 & 22.3 & 24.3 \\
\hline A lot/Completely & 26.8 & 36.5 & 41.2 & 25.4 & 30.4 & 30.5 \\
\hline I don't know & 1.6 & 1.7 & 4.1 & 0.3 & 1.2 & 1.8 \\
\hline Waterpipe smoke can harm children & $N(1493)$ & $N(786)$ & $N(785)$ & $N(1560)$ & $N(863)$ & $N(1561)$ \\
\hline Not at all/Little & 12.2 & 7.3 & 10.3 & 12.1 & 22.2 & 8.2 \\
\hline Somewhat & 13.6 & 9.2 & 8.3 & 13.8 & 13.9 & 10.9 \\
\hline A lot/Completely & 72.4 & 82.8 & 79.9 & 72.8 & 62.7 & 79.4 \\
\hline I don't know & 1.8 & 0.8 & 1.5 & 1.3 & 1.2 & 1.5 \\
\hline $\begin{array}{l}\text { Waterpipe smoking causes fatal lung } \\
\text { disease }\end{array}$ & $N(1465)$ & $N(785)$ & $N(784)$ & $N(1555)$ & $N(853)$ & $N(1563)$ \\
\hline Not at all/Little & 14.4 & 10.5 & 11.0 & 15.2 & 23.6 & 11.1 \\
\hline Somewhat & 21.0 & 16.3 & 11.6 & 23.1 & 19.9 & 18.4 \\
\hline A lot/Completely & 61.2 & 69.8 & 74.6 & 57.9 & 53.6 & 67.1 \\
\hline I don't know & 3.4 & 3.4 & 2.8 & 3.7 & 2.9 & 3.6 \\
\hline Waterpipe smoking causes cancer & $N(1465)$ & $N(785)$ & $N(773)$ & $N(1542)$ & $N(821)$ & $N(1559)$ \\
\hline Not at all/Little & 18.2 & 14.4 & 12.9 & 19.5 & 23.3 & 15.9 \\
\hline Somewhat & 20.7 & 20.9 & 14.2 & 23.4 & 19.9 & 20.1 \\
\hline A lot/Completely & 53.0 & 57.7 & 66.1 & 49.1 & 51.4 & 55.4 \\
\hline I don't know & 8.1 & 7.0 & 6.7 & 8.0 & 5.5 & 8.5 \\
\hline $\begin{array}{l}\text { Waterpipe smoking causes strokes and } \\
\text { heart disease }\end{array}$ & $N(1477)$ & $N(783)$ & $N(778)$ & $N(1545)$ & $N(849)$ & $N(1554)$ \\
\hline Not at all/Little & 20.8 & 14.0 & 14.0 & 21.7 & 28.4 & 16.2 \\
\hline Somewhat & 19.1 & 19.8 & 13.0 & 22.5 & 18.7 & 19.4 \\
\hline A lot/Completely & 52.8 & 58.9 & 65.9 & 48.4 & 47.5 & 56.3 \\
\hline I don't know & 7.3 & 7.3 & 7.1 & 7.3 & 5.4 & 8.0 \\
\hline $\begin{array}{l}\text { Waterpipe smoking during pregnancy } \\
\text { can harm the baby }\end{array}$ & $N(1472)$ & $N(786)$ & $N(776)$ & $N(1550)$ & $N(843)$ & $N(1558)$ \\
\hline Not at all/Little & 11.0 & 6.2 & 10.3 & 10.5 & 20.5 & 7.6 \\
\hline Somewhat & 11.8 & 9.0 & 7.6 & 12.5 & 13.5 & 9.6 \\
\hline A lot/Completely & 71.1 & 82.2 & 78.6 & 71.7 & 62.0 & 77.7 \\
\hline I don't know & 6.1 & 2.5 & 3.5 & 5.4 & 3.9 & 5.1 \\
\hline Waterpipe smoking can kill you & $N(1463)$ & $N(782)$ & $N(766)$ & $N(1544)$ & $N(820)$ & $N(1557)$ \\
\hline Not at all/Little & 26.4 & 23.8 & 18.4 & 29.5 & 32.3 & 24.1 \\
\hline Somewhat & 19.8 & 20.3 & 18.8 & 20.0 & 17.7 & 20.4 \\
\hline A lot/Completely & 47.1 & 48.1 & 57.6 & 42.6 & 44.1 & 48.0 \\
\hline I don't know & 6.8 & 7.8 & 5.2 & 7.9 & 5.9 & 7.5 \\
\hline $\begin{array}{l}\text { Waterpipe smoke causes fatal lung } \\
\text { disease in nonsmokers }\end{array}$ & $N(1487)$ & $N(784)$ & $N(785)$ & $N(1554)$ & $N(862)$ & $N(1556)$ \\
\hline Not at all/Little & 26.0 & 19.5 & 19.5 & 27.2 & 30.6 & 23.4 \\
\hline Somewhat & 20.8 & 22.2 & 17.2 & 22.7 & 18.8 & 21.5 \\
\hline A lot/Completely & 44.9 & 51.1 & 56.9 & 41.7 & 45.7 & 45.9 \\
\hline I don't know & 8.3 & 7.1 & 6.4 & 8.4 & 4.9 & 9.2 \\
\hline $\begin{array}{l}\text { Quitting Waterpipe smoking now } \\
\text { greatly reduces serious risks to your } \\
\text { health }\end{array}$ & $N(1487)$ & $N(787)$ & $N(784)$ & $N(1554)$ & $N(855)$ & $N(1564)$ \\
\hline
\end{tabular}


Table 3. Continued

\begin{tabular}{|c|c|c|c|c|c|c|}
\hline & \multicolumn{2}{|c|}{ Gender } & \multicolumn{2}{|c|}{ Current WTS } & \multicolumn{2}{|c|}{ Ige } \\
\hline & Vale & Female & No & Yes & $18-22$ & $23+$ \\
\hline Not at all/Little & 19.4 & 12.5 & 16.1 & 20.3 & 30.1 & 15.2 \\
\hline Somewhat & 15.3 & 17.3 & 10.6 & 18.3 & 15.7 & 15.5 \\
\hline A lot/Completely & 61.4 & 66.7 & 69.8 & 57.5 & 51.3 & 65.1 \\
\hline I don't know & 4.0 & 3.6 & 3.6 & 3.9 & 2.9 & 4.2 \\
\hline Mean & 4.75 & 5.41 & 5.68 & 4.56 & 3.99 & 5.14 \\
\hline (SD) & (3.14) & $(3.00)$ & (3.17) & $(3.02)$ & $(3.46)$ & (2.99) \\
\hline
\end{tabular}

Figure 2. Perceived relative harm and addictiveness for waterpipe vs cigarettes among university students who are ever waterpipe smokers in five Eastern Mediterranean countries, 2016

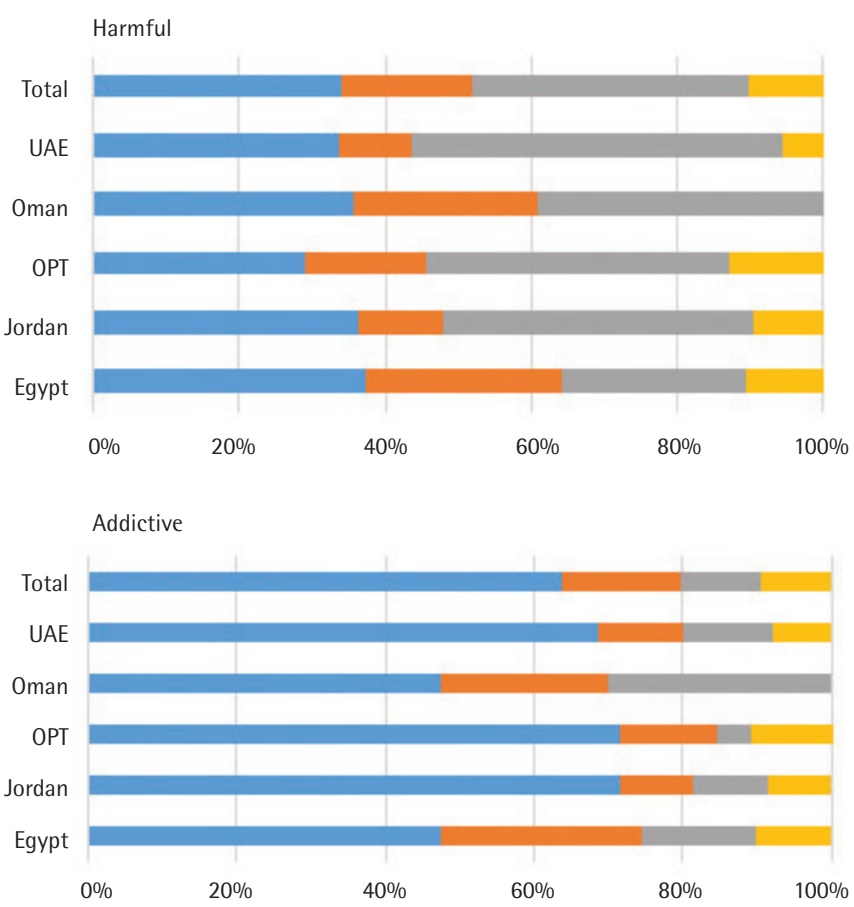

Nicotine Content
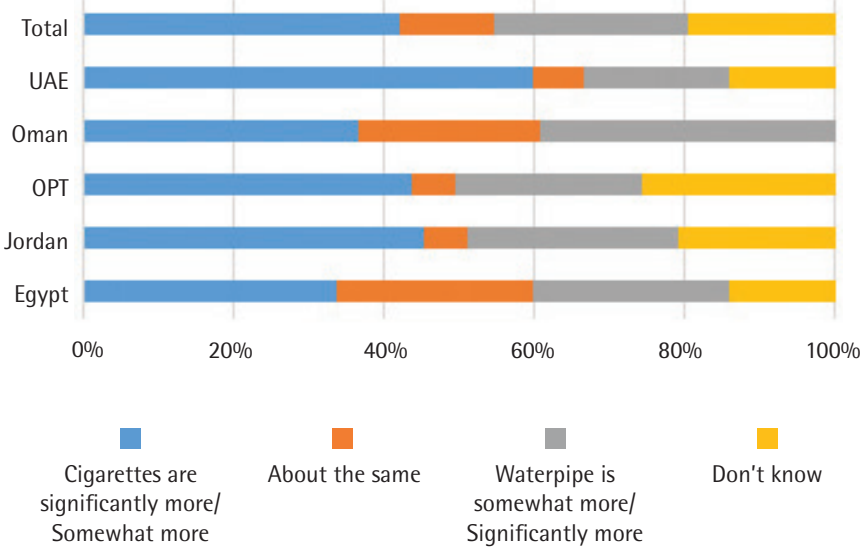

and Palestinian students considered cigarettes more addictive than WTS. Less than a quarter of the students $(26 \%)$ thought that WTS had more nicotine, while $42 \%$ thought that cigarettes had more nicotine. The UAE students compared to those from the other countries had the lowest percentage and thought that WTS had more nicotine compared to cigarettes. Students' perceptions about the harmful effects of cigarettes and WTS were similar: $34 \%$ perceived cigarettes to be more harmful, and $37 \%$ perceived WTS to be more harmful. The lowest percentage (25\%) was that for students from Egypt who thought WTS as less harmful than cigarette smoking, compared with other countries (Figure 2).

Mean harm perception scores were significantly higher among students from OPT $(\mathrm{p}<0.001)$, Jordan $(\mathrm{p}<0.001)$ and UAE $(\mathrm{p}=0.019)$ compared to students from Egypt and Oman after adjusting for age, gender and current WTS. Overall, current WTS students had significantly lower mean harm perception scores compared to non WTS students $(\mathrm{p} \leqslant 0.001)$.

\section{DISCUSSION}

Despite increasing evidence on a WTS epidemic in the EMR, there is generally no parallel public health action taken to tackle this phenomenon ${ }^{14}$. The current study shows that university students in general had low knowledge of WTS harm, with misconceptions about the harmful and addictive effects of WTS. The level of knowledge on harm was lowest among the students from Egypt, despite several original research studies and systematic reviews documenting the harmful effects of WTS on health ${ }^{17-20}$.

An interesting finding was knowledge of the harmful effects that secondhand smoke from WTS has on children and on the fetus of women who smoke during pregnancy. Students perceived WTS as 
less harmful to themselves and to other non-smokers exposed to waterpipe. Another misconception was the belief that WTS is less addictive and contains less nicotine compared to cigarettes. In addition, the students' belief was split between WTS was more harmful compared to cigarettes smoking, WTS had similar harm as cigarettes, and cigarettes were regarded as more harmful in all five countries. Increasing evidence supports that the harmful and addictive effects of WTS are similar to those of cigarette smoking ${ }^{21-23}$. The results of the current study confirm what was reported regarding perception and knowledge in a preliminary qualitative study that was conducted on EMR students through in-depth interviews ${ }^{24}$.

Egypt was found to have a uniquely high percentage of current smokers of waterpipe, cigarettes and dual, in addition to a high level of misconception of the harmful effects of waterpipe. These findings are consistent with prior studies conducted among different populations within Egypt that have indicated the high and increasing prevalence of WTS and the misconception of the harmful effects of waterpipe ${ }^{25,26}$.

There were variations across the five countries with respect to WTS initiation place and company. Jordanian and Palestinian students started WTS at home, whereas Omani, Emirati and Egyptian students more frequently initiated WTS at cafes or restaurants. The previous study explained the influence of smoking parents, especially on their children $^{27-29}$, in addition to the influence of friends and peers ${ }^{29,30}$. Cultural acceptance might explain the high percentage of WTS compared to cigarettes smoking ${ }^{31}$, especially for women ${ }^{32,33}$. The Arab communities are more permissive toward WTS compared to cigarettes, even among conservative communities ${ }^{27,34}$.

Current tobacco policies in the selected countries are generally targeted toward cigarette control, which marginalizes control of other tobacco products including the waterpipe ${ }^{35}$. These countries have approved the World Health Organization Framework Convention on Tobacco Control (WHO FCTC), with the exception of OPT. Still, the enforcement of the WHO FCTC is low in most countries ${ }^{36}$ with more emphasis on text health warnings ${ }^{37}$. Thus, immediate action is needed, similar to cigarette control regulations, targeting bans on advertisement and sales to minors, taxation, warning labels, and smoke-free indoor air policies, to control WTS ${ }^{38}$.

Furthermore, health warning labels on tobacco packages serve as a prominent source of health information for smokers and non-smokers that increases health knowledge and perceptions of tobacco risks. The evidence indicates that comprehensive warnings, specifically pictorial health warnings, may help prevent smoking initiation ${ }^{39}$. The challenge with implementing health warnings for WTS is in its social nature, as it is served in cafes, resturants and homes, and consequently, the smoker does not have direct view of the tobacco packages and labels ${ }^{40}$. Hence, in addition to health warning labels on the waterpipe tobacco packages, labels need to be placed on waterpipe accessories ${ }^{41}$.

Currently, there is limited evidence on the most effective intervention for WTS prevention and cessation. Some studies are showing promising results, especially in increasing the level of knowledge about the harmful effects of WTS ${ }^{42}$. Behavioral cessation interventions based on evidence from cigarette smoking seems to be a good starting point. However, such interventions should take into consideration the cultural and social acceptance of WTS and its intermittent behaviour ${ }^{38}$.

\section{Strengths and limitations}

This WTS study was conducted in five EMR countries using standardised methods on university students. The web-based survey might have some limitations, but it was the method of choice because it requires less time and effort. Further, the web-based surveys have been shown to yield similar response rates compared with more traditional survey modes, and perhaps higher response rates among university students. The study was based on convenience samples so findings may not be generalizable to a broader population. The sample was limited to ever users of WTS and future studies should focus on never users of WTS. The desired sample size was achieved for Egypt, Jordan and OPT, where waterpipe smoking prevalence is higher ${ }^{43}$ and smoking is more socially acceptable $^{24}$ than in Oman and UAE.

\section{CONCLUSIONS}

Misperceptions of waterpipe harm are common 
among university students in the five EMR countries. Immediate public health action is needed, including enforcement of waterpipe tobacco control regulations along with awareness campaigns.

\section{REFERENCES}

1. Akl EA, Gunukula SK, Aleem S, et al. The prevalence of waterpipe tobacco smoking among the general and specific populations: a systematic review. BMC Public Health. 2011;11. doi:10.1186/1471-2458-11-244

2. Ward K, Eissenberg T, Rastam S, Asfar T, Mzayek F, Fouad M. The tobacco epidemic in Syria. Tob Control. 2006;15. doi:10.1136/tc.2005.014860

3. Maziak W, Ward KD, Afifi Soweid RA, Eissenberg T. Tobacco smoking using a waterpipe: a re-emerging strain in a global epidemic. Tob Control. 2004;13(4):327-333. doi:10.1136/tc.2004.008169

4. Azab M, Khabour OF, Alkaraki AK, Eissenberg T, Alzoubi $\mathrm{KH}$, Primack BA. Water pipe tobacco smoking among university students in Jordan. Nicotine Tob Res. 2010;12. doi:10.1093/ntr/ntq055

5. Poyrazoglu S, Sarli S, Gencer Z, Gunay O. Waterpipe (narghile) smoking among medical and non-medical university students in Turkey. Ups J Med Sci. 2010;115. doi:10.3109/03009734.2010.487164

6. Tucktuck M, Ghandour R, Abu-Rmeileh NME. Waterpipe and cigarette tobacco smoking among Palestinian university students: a cross-sectional study. BMC Public Health. 2017;18(1):1. doi:10.1186/s12889-017-4524-0

7. Ghafouri N, Hirsch J, Heydari G, Morello G, Kuo G, Singh R. Waterpipe smoking among health sciences university students in Iran: perceptions, practices and patterns of use. BMC Res Notes. 2011;4. doi:10.1186/1756-0500-4496

8. Kheirallah KA, Alsulaiman JW, Mohammad H AS, Alzyoud S, Veeranki SP, Ward KD. Waterpipe Tobacco Smoking among Arab Youth; a Cross-Country Study. Ethn Dis. 2016;26(1):107-112. doi:10.18865/ed.26.1.107

9. Maziak W,EissenbergT,Rastam S, etal. Beliefs and attitudes related to narghile (waterpipe) smoking among university students in Syria. Ann Epidemiol. 2004;14(9):646-654. doi:10.1016/j.annepidem.2003.11.003

10. Mohammed HR, Zhang Y, Newman IM, Shell DF. Waterpipe smoking in Kuwait. East Mediterr Health J. 2010;16(11):1115-1120.

11. Kheirallah KA AS, Ward KD. Waterpipe Use and Cognitive Susceptibility to Cigarette Smoking Among Never-Cigarette Smoking Jordanian Youth: Analysis of the 2009 Global Youth Tobacco Survey. Nicotine \& Tobacco Research. 2015;17(3):280-284. doi:10.1093/ ntr/ntu 140

12. Warren CW, Jones NR, Eriksen MP, Asma S. Patterns of global tobacco use in young people and implications for future chronic disease burden in adults. Lancet. 2006;367(9512):749-753. doi:10.1016/s0140-6736(06)68192-0

13. Khattab A, Javaid A, Iraqi G, et al. Smoking habits in the Middle East and North Africa: Results of the BREATHE study. Respiratory Medicine. 2012;106 Supplement 2:S16-S24. doi:10.1016/s0954-6111(12)70011-2

14. Maziak W. The waterpipe: time for action. Addiction. 2008;103(11):1763-1767. doi:10.1111/j.13600443.2008.02327.x

15. Maziak W. The waterpipe: an emerging global risk for cancer. Cancer Epidemiology. 2013;37(1):1-4. doi:10.1016/j.canep.2012.10.013

16. Akl EA, Jawad M, Lam WY, Co CN, Obeid R, Irani J. Motives, beliefs and attitudes towards waterpipe tobacco smoking: a systematic review. Harm Reduction Journal. 2013;10:12. doi:10.1186/1477-7517-10-12

17. Gandini S, Botteri E, Iodice S, et al. Tobacco smoking and cancer: a meta-analysis. Int J Cancer. 2008;122(1):155164. doi:10.1002/ijc.23033

18. Akl EA, Gaddam S, Gunukula SK, Honeine R, Jaoude PA, Irani J. The effects of waterpipe tobacco smoking on health outcomes: a systematic review. International Journal of Epidemiology. 2010;39(3):834-857. doi:10.1093/ije/dyq002

19. Akl EA, Jawad M, Lam WY, Co CN, Obeid R, Irani J. Motives, beliefs and attitudes towards waterpipe tobacco smoking: a systematic review. Harm Reduction Journal. 2013;10(1):12. doi:10.1186/1477-7517-10-12

20. Waziry R, Jawad M, Ballout RA, Al Akel M, Akl EA. The effects of waterpipe tobacco smoking on health outcomes: an updated systematic review and meta-analysis. International Journal of Epidemiology. 2017;46(1):3243. doi:10.1093/ije/dyw021

21. Lipkus IM, Eissenberg T, Schwartz-Bloom RD, Prokhorov AV, Levy J. Affecting perceptions of harm and addiction among college waterpipe tobacco smokers. Nicotine Tob Res. 2011;13. doi:10.1093/ntr/ntr049

22. Jackson D, Aveyard P. Waterpipe smoking in students: Prevalence, risk factors, symptoms of addiction, and smoke intake: evidence from one British university. BMC Public Health. 2008;8. doi:10.1186/1471-2458-8-174

23. Aboaziza E, Eissenberg T. Waterpipe tobacco smoking: what is the evidence that it supports nicotine/tobacco dependence? Tobacco Control. 2015;24(Suppl 1):i44-i53. doi:10.1136/tobaccocontrol-2014-051910

24. Salloum RG, Abu-Rmeileh N, Hamadeh R, et al. PolicyRelevant Context of Waterpipe Tobacco Smoking Among University Students in Six Countries Across the Eastern Mediterranean Region: A Qualitative Study. Asian Pacific Journal of Cancer Prevention. 2017;18(9):2533-2540.

25. World Health Organization. Waterpipe tobacco smoking: health effects, research needs and recommended actions by regulators. Geneva: 2005.

26. Egyptian Ministry of Health and Population, World Health Organization. Shisha and smokeless tobacco use among university students in Egypt: prevalence, determinants, 
and economic aspect. http://applications.emro.who. int/dsaf/EMROPUB_2014_EN_1752.pdf?ua=1\&ua=1. Published 2014. Accessed March 13, 2018.

27. Afifi R, Khalil J, Fouad F, et al. Social norms and attitudes linked to waterpipe use in the Eastern Mediterranean Region. Social Science \& Medicine. 2013;98(Supplement C):125-134. doi:10.1016/j.socscimed.2013.09.007

28. Ward KD, Eissenberg T, Rastam S, et al. The tobacco epidemic in Syria. Tobacco Control. 2006;15(suppl 1):i24-i29. doi:10.1136/tc.2005.014860

29. Rice VH, Templin T, Kulwicki A. Arab-American adolescent tobacco use: four pilot studies. Preventive Medicine. 2003;37(5):492-498. doi:10.1016/s0091-7435(03)00175-0

30. Hammal F, Mock J, Ward K, Eissenberg T, Maziak W. A pleasure among friends: how narghile (waterpipe) smoking differs from cigarette smoking in Syria. Tob Control. 2008;17. doi:10.1136/tc.2007.020529

31. Khabour OF, Alzoubi KH, Eissenberg T, et al. Waterpipe tobacco and cigarette smoking among university students in Jordan. The International Journal of Tuberculosis and Lung Disease. 2012;16(7):986-992. doi:10.5588/ijtld.11.0764

32. Almerie MQ, Matar HE, Salam M, et al. Cigarettes and waterpipe smoking among medical students in Syria: a cross-sectional study. The International Journal of Tuberculosis and Lung Disease. 2008;12(9):1085-1091.

33. Maziak W. The waterpipe: time for action. Addiction. 2008;103. doi:10.1111/j.1360-0443.2008.02327.x

34. Dar-Odeh NS, Abu-Hammad OA. The changing trends in tobacco smoking for young Arab women; narghile, an old habit with a liberal attitude. Harm Reduction Journal. 2011;8(1):24. doi:10.1186/1477-7517-8-24

35. Jawad M, El Kadi L, Mugharbil S, Nakkash R. Waterpipe tobacco smoking legislation and policy enactment: a global analysis. Tobacco Control. 2014. doi:10.1136/tobaccocontrol-2014-051911

36. World Health Organization. Tobacco control country profiles. 2013

37. Mostafa A, Kashiwabara M. Tobacco packaging and labelling policies in countries of the Eastern Mediterranean and Western Pacific Regions: Post-deadline assessment of the time-bound measures of WHO FCTC Article 11. Tobacco Prevention \& Cessation. 2016;2(78). doi:10.18332/ tpc/66793

38. Maziak W, Ward KD, Eissenberg T. Interventions for waterpipe smoking cessation. Cochrane Database of Systematic Reviews. 2007 (4). doi:10.1002/14651858.CD005549.pub2

39. Hammond D. Health warning messages on tobacco products: a review. Tobacco Control. 2011; 20(5):327337. doi:10.1136/tc.2010.037630

40. Islam F, Salloum RG, Nakkash R, Maziak W, Thrasher JF. Effectiveness of Health Warnings for Waterpipe Tobacco Smoking among College Students. International Journal of Public Health. 2016;61(6):709-715. doi:10.1007/s00038-016-0805-0

41. Mostafa A, Mohammed HT. Graphic health warnings and their best position on waterpipes: A cross-sectional survey of expert and public opinion. Tobacco Prevention \& Cessation. 2017; 3(116). doi:10.18332/tpc/70873

42. Jawad M, Jawad S, Waziry RK, Ballout RA, Akl EA. Interventions for waterpipe tobacco smoking prevention and cessation: a systematic review. Scientific Reports. 2016;6. doi:10.1038/srep25872

43. Jawad M, Lee JT, Millett C. Waterpipe Tobacco Smoking Prevalence and Correlates in 25 Eastern Mediterranean and Eastern European Countries: Cross-Sectional Analysis of the Global Youth Tobacco Survey. Nicotine \& Tobacco Research. 2016;18(4):395-402. doi:10.1093/ ntr/ntv101
CONFLICTS OF INTEREST Authors have completed and submitted the ICMJE Form for Disclosure of Potential Conflicts of Interest and none was reported.

FUNDING

There was no source of funding for this research.

PROVENANCE AND PEER REVIEW

Not commissioned; externally peer reviewed 